\title{
Perceptibility of schematic face stimuli: Evidence for a perceptual Gestalt
}

\author{
DONALD HOMA, BRAM HAVER, and TERRENCE SCHWARTZ \\ New College, Sarasota, Florida 39578
}

\begin{abstract}
The perceptibility of face, scrambled face, and single-feature stimuli was investigated in three experiments. Stimuli were presented tachistoscopically, followed by a visual noise mask and a forced-choice test of one of three features (eyes, nose, and mouth). In Experiment I, two processing strategies which have been proposed for word perception (involving expectancy and redundancy) were investigated for the stimuli employed here. In Experiments II and III, experimentally induced familiarity was studied for its effect on recognition and perception, and an immediate and delayed perceptual test was employed. Across all three experiments, perception of single-feature and face stimuli were consistently superior to scrambled faces; in Experiment III, differences between single features and faces were eliminated. The effects of perceptual expectancy, internal feature redundancy, familiarity, guessing biases, etc., were shown to be insufficient to account for the superiority of face to scrambled face stimuli. It was argued that the perceptibility of nonredundant features are enhanced when those features are aligned in a well-defined form class. The view that familiarity operates directly on recognitive processes but indirectly on perceptual ones was discussed.
\end{abstract}

The purpose of the present series of experiments was to assess the likelihood that a well-defined form class might facilitate the perceptibility of its constituent features. In experiments involving word or word-like stimuli (e.g.. spelling patterns, syllables, etc.), it has been frequently observed that perceptibility of all letters in a word is greater than perceptibility of a single letter shown in isolation or the same letters scrambled to form an unprounceable nonword (Baron \& Thurston, 1973; Reicher, 1969). Since neither a serial processing model (e.g.. Sperling. 1963), which would predict letter perception to be more accurate than either word or nonword perception. nor a noninteractive parallel processing model (e.g., Rumelhart. 1970), which could predict equivalent performance on single letters and words, can account for this word superiority effect (WSE), recent attempts have focused upon potential methouological problems (Thompson \& Massaro. 1973; Wheeler, 1970) or more complex processing strategies (Smith \& Haviland, 1972) to account for these results.

A somewhat neglected issue is the generalizability of the WSE to other materials, i.e., in general. is the perceptibility of the constituent parts of a stimulus configuration enhanced when those parts are ordered

Requests for reprints should be sent to Donald Homa, Department of Psychology, Arizona State University, Tempe, Arizona 85281 . Experiment III was submitted by B. Haver in partial fulfillment toward a senior thesis at New College, Sarasota, Florida. Portions of Experiment III were reported at the Southeastern Psychological Association in Atlanta, Georgia, March 1975. T. Schwartz is now a graduate student at the University of Oregon. Portions of Experiment I were reported at the Southeastern Psychological Association in Hollywood, Florida. May 1974. or organized in some well-detined pattern? For example, learning of patterns, including words, might be characterized as proceeding initially from an emphasis on individual features to some final learning state where the elements or features are themselves integrated into the overall configuration. ${ }^{1}$ Conceivably, the perceptual processing of overlearned patterns might involve these higher order units, much in the same way that an abstracted prototype (e.g., Posner \& Keele, 1968) functions as a higher order unit for a concept.

The three experiments reported here investigated the processing of schematic face, scrambled face, and single component parts under a variety of conditions. In Experiment I, the likelihood of two processing strategies that have been suggested for word stimuli were tested for the form stimuli employed here. In Experiment II, the effects of experimentally induced familiarity were assessed on the perceptibility of these stimuli. In Experiment III. familiarity was again studied, but a different familiarization procedure was used and a recognition test was included prior to perceptual testing; in addition, the effects of immediate and delayed perceptual testing were explored.

\section{EXPERIMENT I}

Two processing strategies that have been proposed to account for the WSE are relevant to Experiment I: (a) the inference hypothesis (Wheeler, 1970) and (b) the unitization hypothesis (Smith \& Haviland, 1972). Briefly, the inference hypothesis maintains that features are always extracted (Neisser. 1967), but that later feature extractions are somehow guided by the output of earlier features and feature tests. Thus, 
crucial features are inferred from prior information, and this nonrandom extraction process is responsible for the WSE. The unitization hypothesis proposes that expectancies and processing strategies govern the unit of analysis (Aderman \& Smith, 1971) and that words can be processed according to these higher order units (e.g.. spelling patterns). A major distinction between these two processing strategies is that, under the unitization hy pothesis, the stimulus may be segmented prior to analysis, whereas the inference hypothesis implies that facilitation of critical feature tests can occur only during analysis. In order to test these two hypotheses. Smith and Haviland (1972) trained subjects to learn the sequential redundancy of three-letter stimuli. half of which were unpronounceable trigrams (e.g.. rdm) and half, three-letter words (e.g., ram). Since sequential redundancy was also equated for the two types of material, and since subjects were required to learn the letter-by-letter sequential rules for both stimulus types, it was argued that any perceptual superiority for words would rule out the inference hypothesis as an explanation for the WSE. The result was that word perception was not only superior to the nonwords, but the size of the difference did not diminish with 4 days' practice. However, a recent experiment by Mezrich (1973) was able to reverse the WSE under one condition that involved vocalization and a delayed test. and this result seems incompatible with the unitization hypothesis, i.e., the unitization hypothesis must predict that word perception should be either suprior to or at least equivalent to single-letter perception, and that variables operating after presentation of the stimulus (e.g.. vocalization. delaying the test) should be ineffective.

Three basic stimulus types were used in the present study: (a) hand-drawn faces; (b) scrambled faces. with the eyes, nose, and mouth ordered vertically in a consistent but anomalous manner; and (c) single features (eyes, nose, or mouth). Normal and scrambled faces were constructed by randomly selecting from a pool of features containing five variations each of eyes, nose, and mouth, and reassembling these features ${ }^{2}$ in a head outline. Subjects were always tested on a single feature, and responses were indicated by a variant of a forced-choice procedure. In a sense, the experiment bore a sinilarity to that performed by Reicher: here, the single feature, scrambled face, and normal face may possibly function as their word-type counterparts, e.g.. single letter, scrambled letters, and words, respectively. The WSE would be evidenced by demonstrating that any feature embedded in a face is perceived with greater accuracy than any feature which appeared either by itself or in an anomalous face.

A second manipulation provided a test of the relevance of the unitization and inference hypotheses for form stimuli. Each stimulus type was cued on half the trials prior to presentation; thus, the subject knew, on half the trials, what type of stimulus to expect, but no information about the characteristics of the features themselves was given. In other words, only information about the form class, e.g., "you will be shown a face." was given on cued trials. It was felt that only the unitization hypothesis could accommodate a facilitation in performance under cueing. The experiment by Aderman and Smith (1971) demonstrated that expectancies of stimulus types can have a profound effect on the processing of word-like stimuli, although anomalous letter strings were relatively unaffected by this manipulation. It was also felt that the inference hypothesis could not predict any change in performance as a function of cueing. because foreknowledge of the stimulus type provided no information of the feature variations contained in the stimulus, i.e., each of the three feature types (eyes, nose, and mouth) were randomly sampled from a constant pool of five variations, and all feature variations were equally probable and well known to the subject prior to the experiment; hence, inference, at least for the specific variations of the eyes, nose, and mouth, would seem unlikely as a function of cueing for stimulus types.

Finally, the inference hypothesis would seem to predict no difference between normal and scrambled faces, since redundancy among the specific feature variations was always zero, i.e., knowledge about one of the feature variations for, say, the eyes, provided chance predictability for the specific details of the remaining features (nose and mouth). Any facilitation resulting from embedding a feature within a face must be attributable to organizational principles other than internal redundancy.

\footnotetext{
Method

Subjects. The subjects were 24 New College students who were paid $\$ 1.50$ for their services.

Materials. Initially. a pool of approximately 30 features were hand-drawn or traced from various sources (e.g., art books, comic sections, etc.). about 10 of each being variations of eyes, noses, and mouths. From this pool, five variations of each of each feature type were selected and adjusted for size differences. with the added stipulation that each of the five variations were judged to be easily distinguishable from each other.

Two basic types of materials were then constructed, test cards and stimulus cards. There were three test cards, each of which contained the five variations for a particular feature. e.g., a card marked "Eyes" had the five variations of eyes on the reverse side of the card. The features contained on the test cards were Xeroxed copies of the same features which appeared in the stimulus cards. Following presentation of a stimulus, the subject was instructed to turn over the appropriate feature card and to indicate which of the tive variations of a particular feature had been presented. To facilitate scoring. each of the features on a particular test card was associated with a number (1-5), and responses were always in the form of a number.

A total of 90 stimulus cards was constructed, 30 each of faces. scrambled faces. and single features. Each face was a composite of three features. contained within a head outline, subtending a visual angle of about $4^{\circ} 15$ in the vertical and $3^{\circ} 26$ in the horizontal. The
} 


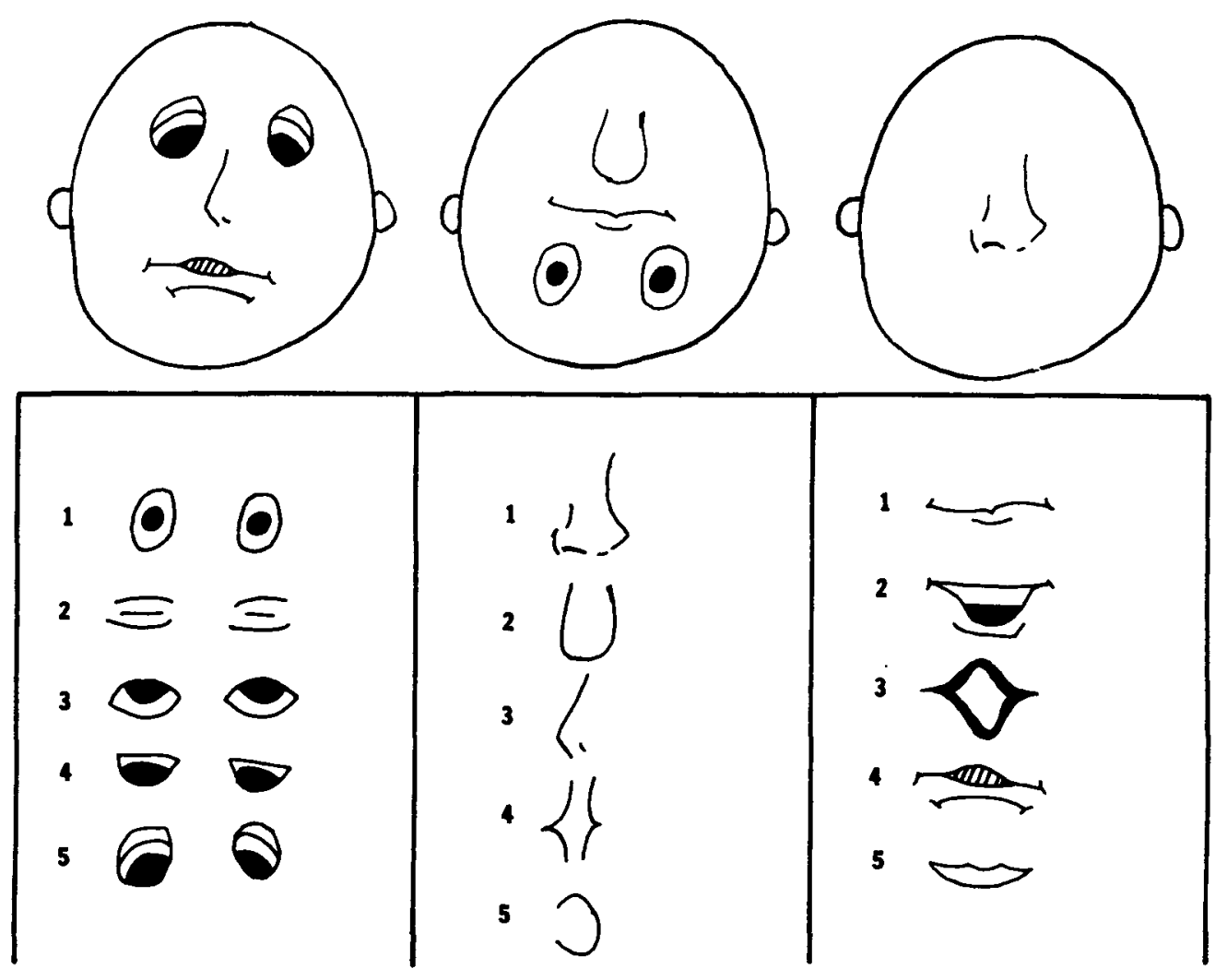

Figure 1. Example of face, scrambled face, and single part stimuli and the three test cards used in Experiment $I$.

30 scrambled faces were constructed by taking one of the face stimuli and realigning its features in the order of nose at top, mouth in the middle, and eyes at the bottom. The set of 30 single features was constructed by placing each of the features in its appropriate location within a head outline. Examples of the stimuli used are shown in the upper panel of Figure 1; the lower panel shows the three test cards and the 15 feature variations.

Procedure. The subject was informed that he would be briefly shown one of three basic stimulus types. faces, scrambled faces, or single features. and that he would always be tested on a single feature. He was further instructed that the features in a scrambled face would appear in the invariant order of nose, mouth, and eyes, from top to bottom; for single features, the feature would appear in its appropriate location within the outline of a head, e.g., eyes always appeared slightly above the center of the head. Prior to presentation, each subject was provided an opportunity to inspect each of the 15 features contained on the test cards. To insure that the subject understood the nature of the task, 10 practice stimuli, composed of the same features as the experimental stimuli but otherwise different from the experimental stimuli in terms of the particular feature combinations used. were presented at an initial exposure duration of $50 \mathrm{msec}$. During these 10 practice trials, each of the six combinations of stimulus type and cueing occurred at least once. The practice trials also provided the opportunity to slightly adjust the exposure duration; if less than 4 of the 10 practice stimuli were answered correctly, the exposure duration for the experimental trials was increased to 60 msec. If less than two errors occurred, the duration was reduced to $45 \mathrm{msec}$.

Following the practice trials. and if the subject had no questions. the presentation of the experimental stimuli was begun. On cued trials, the experimenter stated such and indicated which of the stimulus types was to be shown, e.g., "cued, face." If the trial was uncued, the experimenter stated "uncued." The subject initiated each trial by depressing a response key, which presented a small fixation point, centered in the field, for $2 \mathrm{sec}$. Following offset of the fixation point, the stimulus was shown (for $50 \mathrm{msec}$, unless the adjustment indicated above was used), followed immediately by a 2 -sec visual noise mask. The visual noise mask was composed of a random collection of bits and pieces of the 15 features and covered the entire stimulus configuration. Following offset of the mask, the subject was directed to turn over one of the three test cards before him, and to indicate which of the five variations of that particular feature had been presented. This procedure was repeated for each of the 90 stimuli. Order of presentation of the stimuli was always randomized; a particular random order was used for a block of four subjects and then randomized again for each additional set of four subjects. During the session, the subject sat in a semidarkened room. Each session lasted about $45 \mathrm{~min}$.

Design. The three major variables, stimulus type (face. scrambled face, and single feature), cueing condition (cued and uncued), and feature tested (eyes, nose, and mouth) were factorially combined and manipulated as within-subject variables. For each subject, 45 of the 90 stimuli were cued, and under each cueing condition, each stimulus type occurred equally often, e.g., 15 face stimuli were cued and 15 were uncued. For a given set of 15 stimuli defined by stimulus type and cueing condition, 5 were tested on eyes, 5 on noses, and 5 on mouths. Across all 24 subjects, cach feature occurred equally often within each combination of cueing and stimulus type, and each feature was tested an equivalent number of times. All 30 face and scrambled fact stimuli occurred equally often under cued and uncued conditions.

A three-field tachistoscope (Scientific Prototype, Model GB) was used to present the stimuli.

\section{Results}

For each subject, errors were tabulated for each combination of stimulus type, feature tested, and cueing condition. The mean overall error rate was .54 (chance would be .80 ), with approximately $70 \%$ of the subjects in the error range of $.35-.65$. No subject had 


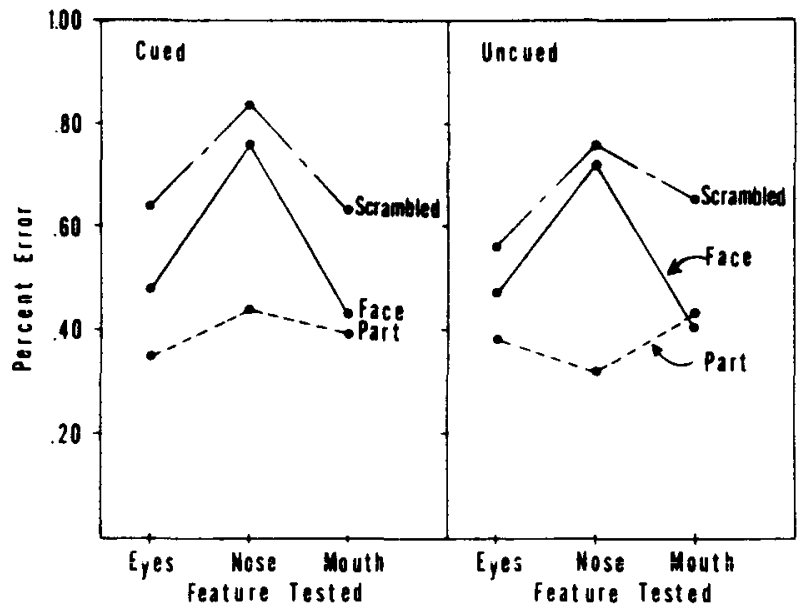

Figure 2. Mean error rate as a function of stimulus type and feature tested, for each cueing condition separately, Experiment I.

an error rate of less than .22 , nor did any subject exceed an error rate of .73. Figure 2 shows the mean error rate, averaged across subjects, as a function of stimulus type and feature tested, for each cueing condition separately. As indicated in Figure 2. performance on stimulus types ordered themselves. with single features, faces, and scrambled faces resulting in error rates of $.385, .543$, and .679 . respectively. An analysis of variance revealed that these differences were highly significant, $F(2.46)=$ $57.10 . \mathrm{p}<.001$ (MSe $=1.37$ ). Only 3 of the 24 subjects made more errors on single features than on faces, and only 3 subjects made more errors on faces than on scrambled faces.

Of the remaining two main variables, the feature tested was highly significant. $F(2,46)=14.14$. $\mathrm{p}<.001(\mathrm{MSe}=2.04)$, whereas the effect of cueing only approached significance. $F(1,23)=2.98$. $\mathrm{p}<.10(\mathrm{MSe}=.95)$. Somewhat unexpectedly, more errors were made under the cued condition (.552) than under the uncued condition (.519).

Judging from the performance on single features. each of the feature types seemed to be of equivalent difficulty, i.e., when eyes, nose, or mouth appeared alone, accuracy of performance did not stray much from the overall error rate for single features (.385). However, for both face and scrambled face stimuli. the eyes and mouth were more accurately perceived. and performance on the nose feature approached random levels. This differential performance on feature tested for the various stimulus types resulted in a significant interaction between stimulus type and feature tested, $F(4,92)=6.53, \mathrm{p}<.001(\mathrm{MSe}=$ 1.56). It might be remembered that the order of features in a scrambled face was, from top to bottom, nose, mouth. and eyes; hence, the difficulty of nose perception for both faces and scrambled faces could not be due to a top-to-bottom scan. This latter result should not be considered at variance with the top-to-bottom sequential scanning found by Smith and Nielsen (1970) in a same-different task involving schematic faces. In their task, the sequential scanning (for different responses) probably involved the comparison of a feature list in memory with a current stimulus; in the present experiment, the scan is assumed to reflect the perception extraction processes, where the order of information gain is not guided by a top-to-bottom process.

Although cueing did not seem to greatly affect overall performance, a mild interaction between cueing and feature tested was evidenced, $F(2.46)=$ $3.78, \mathrm{p}<.05(\mathrm{MSe}=.52)$; this was primarily due to the poorer performance on the nose feature under the cued condition relative to the uncued condition. The interaction between stimulus type and cueing was not significant, $F(2,46)=.18, \mathrm{p}>.20(\mathrm{MSe}=.97)$. The double interaction between stimulus type, cueing, and feature tested also fell far short of significance, $\mathrm{F}(4.92)=1.11, \mathrm{p}>.20(\mathrm{MSe}=.80)$.

\section{EXPERIMENT II}

Although the three stimulus types were readily recognizable as belonging to different form classes, it could be argued that the familiarity of specific feature combinations is a necessary condition for inducing a WSE for pictorial stimuli (features in a face superior to features in isoiation). To assess this possibility, Experiment II investigated the effect of experimentally induced familiarity for face and scrambled face stimuli on perception. This was accomplished by having half the face and scrambled face stimuli made available for study prior to tachistoscopic exposure. The effect of cueing for stimulus types was not investigated further, and all stimuli were presented without cueing.

\begin{abstract}
Method
Subjects. The subjects were 14 New College students, none of whom had participated in Experiment I.

Procedure and Design. Prior to the tachistoscopic trials, each subject was given 30 of the to-be-remembered stimuli and instructed to study them for about $7-8 \mathrm{~min}$. These 30 stimuli consisted of 15 face and 15 scrambled face stimuli, randomly seiected from the stimuli in Experiment I, with the restriction that each of the 15 individual features ( 5 each of eyes, nose, and mouth) occur equally often among the 30 stimuli. The stimuli were contained in a folder, with the 15 face and scrambled face stimuli located on the two inside covers. Each subject was instructed that these and other stimuli not contained in the folder would be shown during the experimental trials, and that familiarization of these stimuli might facilitate performance. The subject was made aware of the different features contained in the stimuli but was otherwise uninstructed during the inspection period. After the inspection period was completed, the experiment proper proceeded as before
\end{abstract}

\section{Results}

The mean error rate for each stimulus type is shown in Figure 3 as a function of feature tested and 
familiarity. The overall error rate for single features, faces, and scrambled faces mirrored the results of Experiment I, with single features having the lowest error rate (.395), scrambled faces the highest (.707), and normal faces intermediate (.493). An analysis of variance with stimulus type (face vs. scrambled) and familiarity as main variables revealed that face stimuli were perceived more accurately than scrambled faces, $F(1,13)=26.34, p<.01(\mathrm{MSe}=5.49)$, but neither the effect of familiarity, $F(1,13)=1.49, p>.20$ $(\mathrm{MSe}=1.73)$, nor the interaction of stimulus type with familiarity, $F(1,13)=.16, p>.20(\mathrm{MSe}=$ 3.95). was significant. The overall error rates for familiar and unfamiliar face stimuli was .500 and .486, respectively; for scrambled face stimuli, these values were .729 and .686 .

A second analysis was performed on stimulus type (face, scrambled face, and single feature) and feature tested. with the variable of familiarity ignored. This analysis essentially replicated the results of Experiment I, with stimulus type and feature tested highly significant, $\mathrm{F}(2,26)=40.19(\mathrm{MSe}=2.66)$ and $\mathrm{F}(2.26)=10.37(\mathrm{MSe}=3.33)$, both $\mathrm{p}<.01$; the interaction of stimulus type with feature tested was also significant, $F(4,52)=2.85, \mathrm{p}<.05(\mathrm{MSe}=$ 2.19). and again reflected the uniform performance of single features when shown in isolation but not when embedded in face and scrambled face stimuli. Thus, the second experiment gave little indication that experimentally induced familiarity, as manipulated here, had any effect.

\section{EXPERIMENT III}

Although familiarity had a minimal effect in Experiment II, it could be argued that the manipulation was too weak for familiarity influences to be revealed. If this were true, it should not obscure the primary finding that the perceptibility of features within faces were enhanced relative to those features within scrambled faces. Nonetheless, Experiment III reinvestigated the effects of experimentally induced familiarity on perception under more convincing and stringent criteria: (a) familiarity was manipulated under five different training levels rather than the familiar-unfamiliar dichotomy in Experiment II, and (b) a recognition forced-choice test preceded the perceptual test of these stimuli. If increasing the number of training trials systematically increases recognition performance but is ineffective in facilitating the identification of tachistoscopically presented stimuli, then the view that familiarity affects memorial but not perceptual processes would be strengthened.

Another manipulation in Experiment III was the use of an immediate and delayed perceptual test. In both Experiments I and II, it could be argued that the slight delay between stimulus offset and turning over

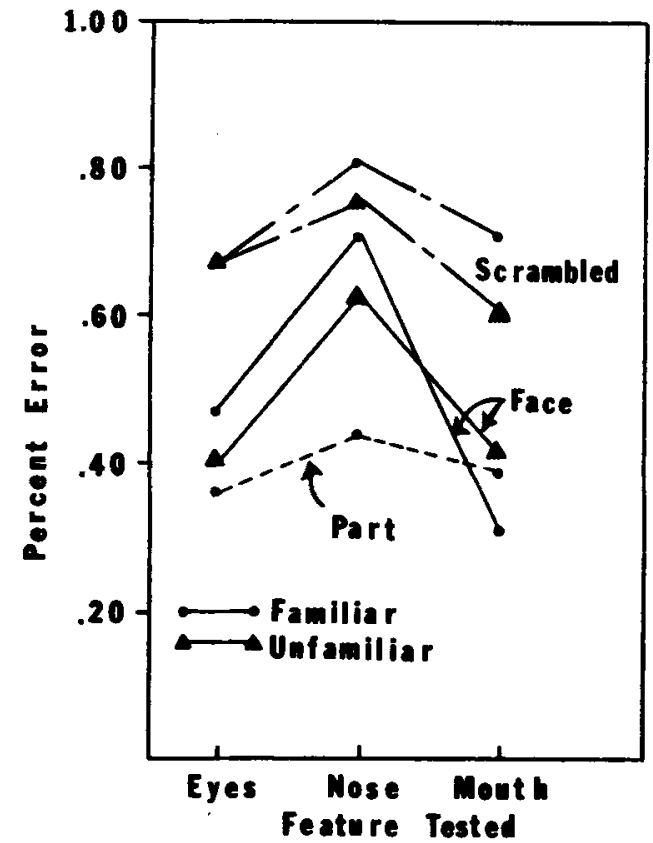

Figure 3. Mean error rate as a function of stimulus type, feature tested, and familiarity, Experiment II.

of the feature test card allowed for the forgetting of information extracted during the stimulus presentation. In fact, if forgetting was greater for scrambled than for normal face stimuli, then results similar to those found in Experiments I and II could have resulted. Should the relative performance for each of the stimulus types be maintained under immediate and delayed testing, then differences between scrambled and normal face stimuli would not be attributable to differential forgetting but to differences in perceptual processing efficiency.

\section{Method}

Subjects. A total of 50 New College students. 25 in each of the immediate and delayed perceptual test conditions. were paid $\$ 3$ for their participation. None of the subjects had been in either Experiment I or Experiment II.

Procedure. Each subject participated in three, distinct phases of the experiment, an initial study-familiarization phase, followed by a forced-choice recognition test, followed by perceptual testing.

In the familiarization phase, a study deck consisting of 264 stimuli was presented in a flash-card manner for about $5 \mathrm{sec}$ each. The subject was instructed to simply observe the stimuli carefully and to "try to remember each face and scrambled face so that you can recognize it later." The study deck was composed of 24 distinct face and 24 distinct scrambled face stimuli, 6 each of which occurred 12 times in the study deck, 6 each which occurred 6 times. 6 each which occurred 3 times, and 6 each which occurred just once. The study deck of 264 stimuli was presented in a random order, with the restriction that no two identical face or scrambled face stimuli appear consecutively. Multiple copies were made by Xeroxing face and scrambled face stimuli employed in the later perceptual test.

Following the study phase, each of the $\mathbf{4 8}$ distinct stimuli was paired with a foil of the same stimulus class. e.g., an old face with a new face. The $\mathbf{4 8}$ stimulus pairs were presented in a random order, and the subject was instructed to indicate which of the two stimuli on each card was old, i.e., had appeared in the study deck. Subjects 
indicated their choices by verbally stating whether the old stimulus was on the left or the right side of the recognition card. There was an equal probability of an old stimulus appearing on the left or right side of the card for both face and scrambled face tests.

The perceptual phase was begun following completion of the recognition test. For subjects in the immediate condition, the sequence of events on each trial was as follows: a small fixation point appeared for about $2 \mathrm{sec}$, followed by the stimulus, followed by a masking field with the five variations of the feature being tested aligned vertically to the left of the masking field. The masking field and test alternatives remained on for $8 \mathrm{sec}$. The procedure for the subjects in the delayed perceptual test condition was identical to that employed in Experiments I and II, and differed from the immediate subjects only in that the masking field appeared by itself, and subjects were required to turn over one of the three feature test cards at the time of test. If there were no questions following instructions for the perceptual phase, subjects were allowed to inspect the feature variations on each of the three test cards.

Two blocks of 10 practice trials were then presented, with the restriction that face, scrambled face, and single-feature stimuli occur at least three times and that each feature (eyes, nose, and mouth) be tested about equally often. Because pilot testing had indicated that the immediate test condition might be more difficult than delayed testing, all stimuli were initially presented for $60 \mathrm{msec}$. If more than eight errors occurred in either block of 10 practice trials, the exposure duration was increased by $5 \mathrm{msec}$; if less than four errors occurred in either block, the duration was decreased by $5 \mathrm{msec}$.

Following the practice trials, presentation of the 90 experimental stimuli was begun, 30 each of face, scrambled face, and single-feature stimuli. For the face and scrambled face stimuli, 6 had occurred 12 times in the study deck, 6 had occurred 6 times, 6 had occurred 3 times, 6 had occurred once, and 6 had never been presented. Within each subset of 6 stimuli, the eyes, nose, and mouth features were tested exactly twice. The 90 experimental stimuli were presented in one of three random orders; within each block of 30 stimuli, face, scrambled face, and single-feature stimuli occurred approximately equally often.

Design. A Latin square was used to determine the familiarity level for each of the face and scrambled face stimuli. The 30 face stimuli which appeared during the perceptual phase were initially grouped into five subsets (A, B, C, D, and E) of six stimuli each. For 5 of the 25 subjects in each of the immediate and delayed perceptual test conditions, the A subset appeared 12 times during

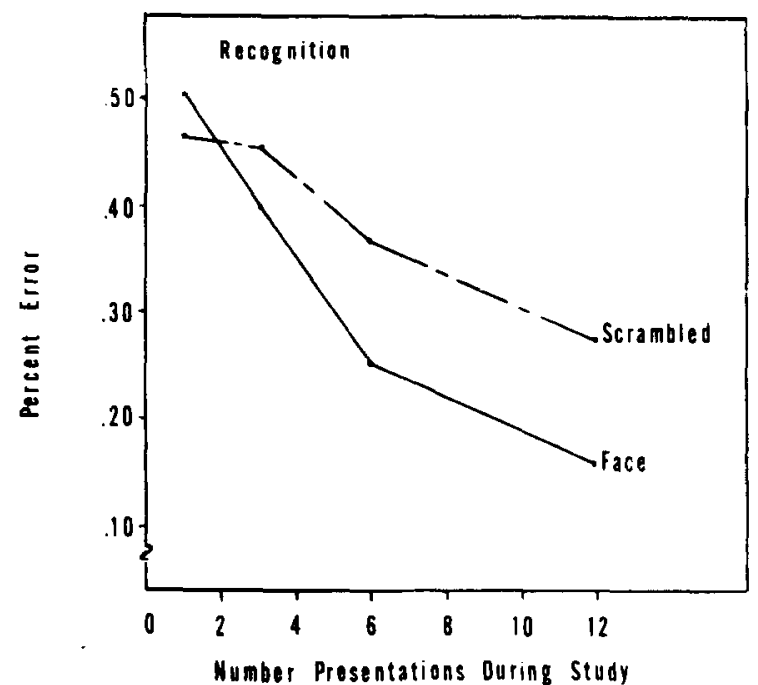

Figure 4. Mean error rate on the recognition test for face and scrambled fact stimuli as a function of number of training presentations, Experiment III. familiarization training, the B subset appeared 6 times, the $C$ subset appeared 3 times, the $D$ subset once, and the $E$ subset 0 times. The five subsets were then rotated across blocks of five subjects so that each stimulus would appear equally often as a 0,1 . 3 . 6 . and 12 familiarization stimulus. An identical balancing procedure was used for the 30 scrambled face stimuli.

In order to enhance the random assignment of an old stimulus with a foil on the recognition test, each of the 30 face and scrambled face stimuli was paired randomly with one of four different foils. This resulted in four different recognition test decks of 60 stimuli; for each subject, the 12 stimuli which had received no familiarization training were then deleted from the deck, leaving the 48 recognition test cards. Subjects from both the immediate and delayed perceptual conditions were then randomly assigned one of the four recognition test decks. Across the four test decks, each of the 30 face and scrambled face stimuli appeared equally often as an old stimulus on the left and right side of the card.

For the perceptual test, approximately equal numbers of subjects were assigned randomly to one of three random-order presentations of the 90 stimuli and to one of three test orders. The test orders differed only in that, for about one-third of the subjects, a particular face or scrambled face was tested on the eyes feature, for another third of the subjects, the nose feature was tested, and for the remaining subjects, the mouth feature was tested. Across subjects, then. each of the face and scrambled face stimuli was tested approximately equally often on each of the three features.

Each of the three phases of the experiment occurred in the same room. Following the study and recognition phase, the room was darkened and the subject was allowed to dark-adapt for about 2 min prior to perceptual testing. Each session lasted about $2 \mathrm{~h}$.

\section{Results}

Recognition testing. A three-way analysis for a mixed design was computed, with stimulus type (face and scrambled) and number of training presentations (1. 3,6 and 12) as within-subject variables and delay of the later perceptual test (immediate and delayed) as the between-subject variable. Since the delay variable pertained only to the later perceptual test, differences on the recognition test should reflect random sampling error. In fact, the effect of delay was not significant, $F(1,48)=.42(\mathrm{MSe}=1.16)$, nor did it significantly interact with either stimulus type, $\mathrm{F}(1.48)=.40(\mathrm{MSe}=1.62)$, or number of presentations, $F(3,144)=1.22(\mathrm{MSe}=1.40)$. $\mathrm{p}>.20$ for all three cases. Figure 4 shows the mean error rate on the recognition test for face and scrambled face stimuli as a function of number of training presentations, averaged across subjects and the two delays.

The analysis revealed that the main effects of number of presentations, $F(3,144)=36.83, \mathrm{p}<.001$ $(\mathrm{MSe}=1.40)$, and stimulus type, $\mathrm{F}(1,48)=8.43$. $\mathrm{p}<.01$ (MSe $=1.62$ ), were both highly significant. The Stimulus Type by Number of Presentations interaction was also significant, $F(3,144)=3.64$, $\mathrm{p}<.03(\mathrm{MSe}=1.32)$.

Individual subject data also reflected these results; excluding ties, about $47 \%$ of the subjects made more recognition errors on face than on scrambled face stimuli for 1 and 3 presentations combined, whereas only $29 \%$ made more face stimuli errors for 6 and 12 presentations. 


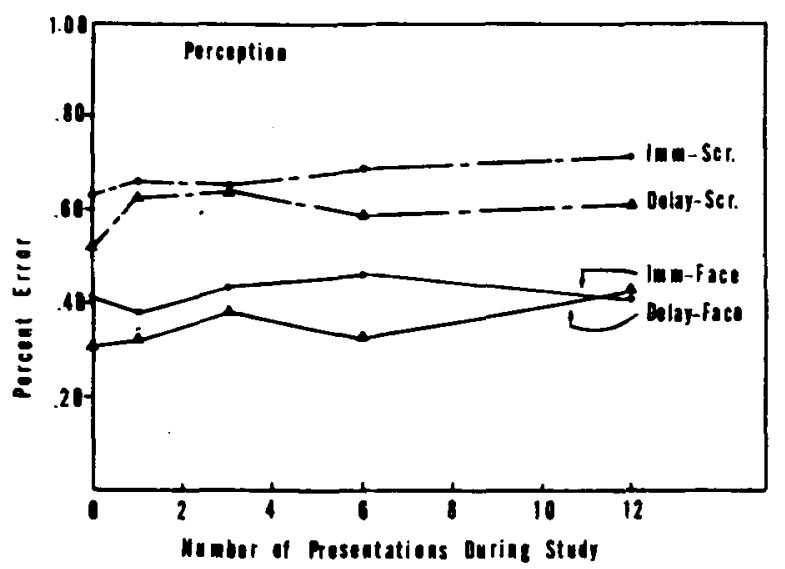

Figure 5. Mean error rate for face and scrambled face stimuli as a function of number of training presentations, immediate and delayed perceptual test, Experiment III.

In summary, the effect of number of presentations dramatically improved recognition of both face and scrambled face stimuli, with face stimuli benefiting more by this manipulation than scrambled faces.

Perceptual testing as a function of familiarization. To determine the influence of familiarization training on perception. separate analyses were computed for immediate and delayed perceptual testing. For each analysis, the variables were number of presentations $(0,1,3,6$, and 12) and stimulus type (face and scrambled). Figure 5 shows the mean error rate for face and scrambled face stinuli as a function of number of study presentations for immediate and delayed testing.

For the immediate test, only the effect of stimulus type was significant, $F(1,24)=99.72, p<.001$ (MSe $=1.42$ ). The effect of number of presentations on perception. $F(4,96)=.73(\mathrm{MSe}=1.50)$, and the Stimulus Type by Number of Presentations interaction, $F(4,96)=.53(\mathrm{MSe}=1.38)$, both failed to reach significance, $\mathrm{p}>.20$ in each case.

For the delayed test, the effect of stimulus type was again significant, $F(1,24)=143.97, \mathrm{p}<.001(\mathrm{MSe}$ $=.94)$. Although the Stimulus Type by Number of Presentations interaction was again insignificant, $F(4,96)=.84, p>.20(\mathrm{MSe}=1.25)$, the main effect of presentations was marginally significant, $F(4,96)$ $=2.66, \mathrm{p}<.05(\mathrm{MSe}=1.36)$. In the latter case, the significance is partly due to the unexpectedly better performance on the scrambled face stimuli for no previous familiarization training.

Overall, these rsults lend little support for the hypothesis that familiarization training affects perception. In the one anomalous case (scrambled face stimuli, delayed test), the effect is actually opposite of what might be expected had it been hypothesized that increased familiarity facilitates perception. Even here, however, the effect was small and may reflect a chance occurrence.
Perceptual testing for stimulus types. An analysis was computed for the immediate and delayed conditions separately, for the variables of stimulus type (face, scrambled, and single parts) and feature tested (eyes, nose, and mouth). Figure 6 shows the mean error rate for stimulus type and feature tested for immediate and delayed testing separately.

For the immediate test, the effect of stimulus type, $F(2,48)=41.16(\mathrm{MSe}=4.55)$, feature tested, $F(2,48)=12.78$ (MSe $=3.95$ ), and the Stimulus Type by Feature Tested interaction, $F(4,96)=18.12$ $(\mathrm{MSe}=2.34)$, were all highly significant, $\mathrm{p}<.001$ in each case.

For the delayed test, a similar patterning of results was obtained. The effect of stimulus type, $F(2,48)=$ $75.98(\mathrm{MSe}=2.21)$, feature tested, $F(2,48)=14.27$ $(\mathrm{MSe}=4.11)$, and the Stimulus Type by Feature Tested interaction, $F(4,96)=10.06(\mathrm{MSe}=3.02)$, were again highly significant, $\mathrm{p}<.001$ in each case.

A number of other results were also of interest: (a) Performance on the delayed test was actually superior to that under an immediate one (error rates of .425 vs. .487 , respectively), so that facilitation, rather than forgetting, resulted by the slight delay of the perceptual test; (b) the effect of the delayed perceptual test was to enhance uniformly the performance of all stimulus types by about $5 \%$; and (c) differences between face and single part stimuli was essentially absent under both an immediate $(.415$ vs. .377) and delayed (.352 vs. .328) test. In neither case was the slight $2.5 \%-4.0 \%$ difference in favor of single parts significant, $\mathrm{p}>.15$.

The last result is perhaps the most interesting. Here, the effect of familiarity was ineffective in improving the perceptibility of face stimuli; yet, the overall differences between face and single part stimuli was eliminated for the first time in these experiments. It may be that familiarity affects perception in an indirect manner; specific stimuli, which have been highly familiarized (as indexed by

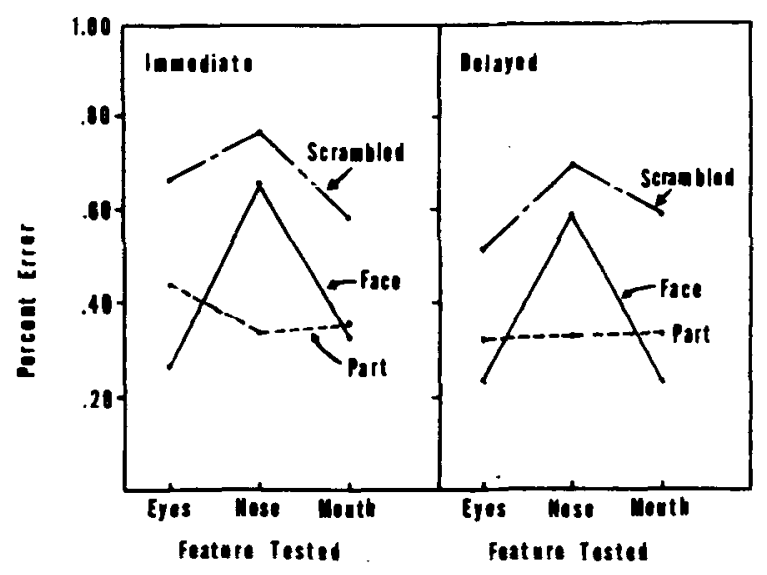

Figure 6. Mean error rate for stimulus type and feature tested for immediate and delayed perceptual test, Experiment III. 
Table 1

Mean Error Rate for Face, Scrambled, and Single Part Stimuli for Each of the Three Experiments

\begin{tabular}{lccc}
\hline $\begin{array}{l}\text { Stimulus } \\
\text { Type }\end{array}$ & I & $\begin{array}{c}\text { Experiments } \\
\text { II }\end{array}$ & III \\
\hline Part & .385 & .395 & .352 \\
Face & .543 & .493 & .384 \\
Scrambled & .679 & .707 & .632 \\
Diff: P - F & -.158 & -.098 & -.032 \\
$\quad$ S - F & +.136 & +.214 & +.248 \\
\hline
\end{tabular}

the recognition test), are perceived no better than their novel counterparts. However, as a form class, the influence of familiarity seemed to be facilitative, enhancing primarily the face stimuli. A comparison of the error rates for each of the three experiments is shown in Table 1, where the immediate and delayed tests for Experiment III are averaged. If the three experiments are roughly viewed as proceeding from a zero to intermediate to high degree of familiarization training (average viewing time per stimulus was about $15 \mathrm{sec}$ in Experiment II and upwards of $30-60 \mathrm{sec}$ in Experiment III for the stimuli presented 6 and 12 times during the study phase), then the superiority of single part stimuli to face stimuli is seen to dminish accordingly (from about $16 \%$ to $10 \%$ to $3.5 \%$ ). This is accomplished by an improvement in the perceptibility of face stimuli while the single part and scrambled face stimuli remained relatively unchanged. The indirect influence of familiarity on the perceptibility of face stimuli may be more complex than indicated, since the improved performance for face stimuli is confined mostly to the eye and mouth features; averaged across immediate and delayed testing, eyes are actually perceived more accurately (by about $15 \%$ ) when embedded in a face than when shown in isolation.

\section{DISCUSSION}

A number of conclusions would seem warranted by the results of the present experiments: (a) The perceptibility of the constituent parts of a stimulus configuration can be enhanced when the parts are arranged within a well-defined pattern; (b) neither perceptual set (or expectancy) nor the internal redundancy of a pattern is a prerequisite for the enhanced perceptibility of features within a well-defined configuration: and (c) familiarity may have a profound facilitative influence on the recognition of stimuli, but little direct effect on the perceptibility of these same stimuli.

The enhanced performance on face stimuli relative to scrambled face stimuli was reliably found across all three experiments. By controlling exposure time, equating guessing bias for stimuli via a variant of the forced-choice procedure (e.g., Reicher, 1969), by eliminating internal redundancy (feature variations within a face and scrambled faces were not predictable from each other) and feature location uncertainty (eyes, nose, and mouth appeared in an invariant order for both faces and scrambled faces) as a potential cue for improving guessing strategies, and by demonstrating that the patterning of results was obtainable under immediate and delayed testing, it seems likely that the results were due to perceptual, and not memorial, effects. In fact, the use of a delayed perceptual test actually improved the performance of all stimulus types, although the patterning of results was apparently unaffected. Experiments which have employed a delayed test following tachistoscopic exposure of word and lettered stimuli have also found that performance is more likely to be improved rather than retarded (Mezrich, 1973; Wheeler, 1970). What seems likely is that, rather than providing an opportunity for the forgetting of stimulus material, the delay between stimulus offset and test allowed for the continued processing or synthesization of the extracted information (Neisser, 1967).

It is interesting to contrast performance on the stimuli employed here with those involving linguistic stimuli, e.g., single letter, words, spelling patterns, nonwords, etc., and to ask whether the variables likely to be effective for word perception have an analogue for form perception. The variables which seem to be of prime importance for determining a WSE are sequential redundancy (Thompson \& Massaro, 1973; Wheeler, 1970) and/or expectancy of the form class of the stimulus to be presented (Aderman \& Smith, 1971). For form stimuli, it might have been anticipated that the effective variables would include the internal redundancy of features within the pattern, and again, expectancy of the form class. What we had originally anticipated was that degree of internal redundancy would interact with stimulus type, but that, at zero levels of redundancy, no differences between face and scrambled faces would be evidenced. However, by consistently finding that a face superiority resulted at zero redundancy, it was concluded that feature inference, based along principles of redundancy, was likely to lead to erroneous conclusions for form stimuli. Furthermore. the failure to obtain an effect of expectancy of form class seemed to preclude any explanation of the perceptual facilitation for face stimuli that was based on principles of perceptual set. Two conclusions which seem forced by these results are: (a) Theories of visual perception which are based on results from studies of linguistic material are inappropriate, since the functional variables for linguistic and form stimuli are likely to be different; and (b) a hypothesis of a perceptual Gestalt must be seriously entertained for well-defined form stimuli, where a perceptual Gestalt is defined as the enhanced perceptibility of the constituent parts due to the general form class of the 
stimulus. A corollary would be that the positive influence of the form class on perception must occur following some initial feature extractions and feature tests, since prior information about the form class (cueing) was relatively ineffective. Perhaps feature extractions become more selective only after a decision about the form class has been determined. In effect. a low-level categorization response may be processed prior to full feature identification. Ingling (1972) has provided some support for the view that stimulus categorization can occur prior to stimulus identification; for a perceptual Gestalt to function effectively on its constituent parts, it may also be necessary that a decision about the form class precede feature identification.

The present results might also be contrasted with those of Biederman (1972) and Biederman, Glass, and Stacy (1973). In these experiments, it was demonstrated that object identification was facilitated when these objects were embedded in coherent real-world scenes compared to jumbled versions of these scenes. It was concluded that the contextual information provided by the coherent scenes facilitated the perceptual identification of the target objects, perhaps by providing for the formation of a schema (Bartlett, 1932). Evidence that both object and schematic information might be simultaneously processed from a scene was provided by Biederman, Rabinowitz, Glass, and Stacy (1974). In their experiment, the ability to characterize a scene was facilitated by increases in exposure duration for coherent scenes but not for jumbled ones; in contrast, object identification increased for both types of scenes across the same exposure durations. Two important differences between Biederman's work and the present study are worth noting, however. First, the target "objects" (eyes, nose, and mouth) in our experiments were always present in both faces and scrambled faces on each trial. What varied was the particular form each feature could assume. Thus, determining whether a fire hydrant was contained in a real-world scene might involve different processes than determining the particular type of the eyes that was contained in a normal face. In our experiments, subjects could not use contextual information to guess whether each face or scrambled face was likely to contain the eyes feature, since eyes were contained in all faces and scrambled faces. Secondly, we were able to contrast performance for a single feature shown in isolation with that feature embedded in a "coherent" or "scrambled" scene. Since single-feature presentation eliminates contextual information except for the critical feature, a control for the effect of context is possible.

The results of Experiment III provide some insight into the role of learning on perception. Consistent with previous experiments which have investigated the frequency-familiarity relationship (Arnoult, 1956;
Noble, 1954), we were able to show that experimentally induced familiarity had a sizable influence on face and scrambled face recognition, with the face stimuli especially profiting by this manipulation. However, no perceptual differences were obtained for those stimuli which had been highly familiarized with those that were either low in familiarity or entirely novel. Still, the overall perceptual differences between face and single-feature stimuli were eliminated in this experiment, although the complex interaction between feature tested and stimulus type was found in this experiment, as it was in Experiments I and II. We would like to speculate that increasing familiarization of a stimulus subset enhances the perceptual codability of all stimuli which belong to that form class, perhaps by modifying an essentially serial, feature-by-feature extraction process to one that is increasingly parallel. However, this hypothesis would be consistent only for the results of the face stimuli, since scrambled face stimuli were largely unaffected by this manipulation. Either the form class must contain a minimum amount of previously learned structure (which would be true for faces but not for scrambled ones), or alternatively, the scrambled face stimuli required utilization of schematic information which was competitive with the fact stimuli.

Finally, a true WSE may have been obtained for highly familiarized face stimuli, since the perceptibility of the eyes feature was substantially superior for face than for single-feature stimuli. If familiarization can bias perceptual encoding, it would seem to do so for the eyes and mouth features at the relative expense of the nose feature. It is noteworthy that single features, in isolation, were equally perceptible. Perhaps for face stimuli, attentional demands in perceptual processing are increasingly drawn to the eyes and mouth. The consistently poor performance on the nose feature, given that it was centrally located in the visual field and of equal discriminability when shown in isolation, would be consistent with this view.

\section{REFERENCES}

Aderman, D., \& Smith, E. E. Expectancy as a determinant of functional units in perceptual recognition. Cognitive Psychology. $1971,2,117-129$.

Arnoult, M. D. Familiarity and recognition of nonsense shapes. Journal of Experimental Psychology. 1956. 51. 209.276.

Baron, J., \& ThuRston. I. An analysis of the word-superiority effect. Cognitive Psychology. 1973. 4. 207-228.

Bartlett. F. C. Remembering: $A$ study in experimental and social psychology. Cambridge: Cambridge University Press, 1932.

Biederman, I. Perceiving real-world scenes. Science, 1972, 177. 77-80.

Biederman, I., Glass, A. L., \& Stacy, E. W., JR. Searching for objects in real-world scenes. Journal of Experimental Psichology, 1973, 97, 22-27. 
Biederman, I., Rabinowitz, J. C., Glass, A. L., \& Stacy, E. W. JR. On the information extracted from a glance at a scene. Journal of Experimental Psychology, 1974, 103. 597-600.

Bower, T. G. R. Development in infancy. San Francisco: Freeman. 1974.

Hubel. D. H., \& WIESEL, T. N. Receptive fields of single neurones in the cat's striate cortex. Journal of Physiology, 1959. 148. 574-591.

INGLING, N. W. Categorization: A mechanism for rapid information processing. Journal of Experimental Psychology, 1972, 94. 239-243.

Mezrich. J. J. The word superiority effect in brief visual displays: Elimination by vocalization. Perception \& Psychophysics. 1973, 13, 45-48.

NeIsSER, U. Cognitive psychology. New York: Appleton-CenturyCrofts, 1967.

Noble, C. E. The familiarity-frequency relationship. Journal of Experimental Psychology, 1954, 47, 13.16.

Posner, M. I., \& KeEle, S. W. On the genesis of abstract ideas. Journal of Experimental Psychology, 1968, 77, 353-363.

ReIcher, G. M. Perceptual recognition as a function of meaningfulness of stimulus material. Journal of Experimental Psychology, 1969, 81, 275-280.

RumelhaRT. D. E. A multicomponent theory of the perception of brietly exposed visual displays. Joumal of Mathematical Psychology, 1970, 7, 191-218.

Smith. E. E., \& Haviland, S. E. Why words are perceived more accurately than nonwords: Inference vs. unitization. Journal of Experimental Psychology, 1972, 92, 59-64.
Smith, E. E., \& Nielsen. G. D. Representations and retrieval processes in short-term memory: Recognition and recall of faces. Journal of Experimental Psychology, 1970, 85. 397-405.

SPERLing. G. A model for visual memory tasks. Human Factors. 1963, 5, 19-31.

Thompson. M. C., \& Massaro, D. W. Visual information and redundancy in reading. Journal of Experimental Psychology. 1973. 98, 49-54.

WhEELER, D. D. Processes in word recognition. Cognitive Psychology, 1970, 1. 59-85.

\section{NOTES}

1. Developmental research has suggested that, under some conditions, a single component of a stimulus may dominate the viewing of a pattern: "It has been found, for example, that young infants around six weeks of age are as likely to smile at two dots as at a real face with two eyes. implying that all of the other information in a real face is not registered by the baby ... Gradually, over the next three months, the overall contour of a face. the presence of a mouth, eyebrows, and so forth becomes important" (Bower. 1974, p. 128).

2. The designation of eyes, nose, and mouth as features is intended for expository purposes only, and does not imply that these features function as feature detectors (Hubel \& Wiesel. 1959) in the visual system.

(Received for publication May 12, 1975; revision accepted July 18,1975 . 